\title{
In Situ Growth and Characterization of Metal Oxide Nanoparticles within Polyelectrolyte Membranes
}

\author{
John Landers ${ }^{+}$, Jonathan Colon-Ortiz ${ }^{+}$, Kenneth Zong, Anandarup Goswami, Tewodros Asefa, \\ Aleksey Vishnyakov, and Alexander V. Neimark*
}

\begin{abstract}
This study describes a novel approach for the in situ synthesis of metal oxide-polyelectrolyte nanocomposites formed via impregnation of hydrated polyelectrolyte films with binary water/alcohol solutions of metal salts and consecutive reactions that convert metal cations into oxide nanoparticles embedded within the polymer matrix. The method is demonstrated drawing on the example of Nafion membranes and a variety of metal oxides with an emphasis placed on zinc oxide. The in situ formation of nanoparticles is controlled by changing the solvent composition and conditions of synthesis that for the first time allows one to tailor not only the size, but also the nanoparticle shape, giving a preference to growth of a particular crystal facet. The high-resolution TEM, SEM/ $E D X, U V$-vis and XRD studies confirmed the homogeneous distribution of crystalline nanoparticles of circa $4 \mathrm{~nm}$ and their aggregates of 10-20 nm. The produced nanocomposite films are flexible, mechanically robust and have a potential to be employed in sensing, optoelectronics and catalysis.
\end{abstract}

$T_{\text {here is a tremendous demand in developing novel polymer }}$ composites that contain an inorganic phase with particular interest towards the inclusion of semiconducting metal oxide nanoparticles (MONP). Doing so endears the composite material to applications in heterogeneous catalysis, ${ }^{[1-3]}$ sensors ${ }^{[4]}$ for both biological compounds ${ }^{[5]}$ and chemical warfare agents $i^{[6,7]}$ actuators, ${ }^{[8]}$ optoelectronics, ${ }^{[9,10]}$ energy storage ${ }^{[11]}$ and chemical protection. ${ }^{[12]}$ In this study, we fabricate MONP-polyelectrolyte nanocomposites and investigate the conditions that lead to the in situ growth of MONP within hydrated polyelectrolyte films impregnated by aqueous solutions of metal salts. By invoking consecutive reaction schemes, the metal cations are converted to MONP within the polyelectrolyte matrix. Nafion serves as the archetype polyelectrolyte, due to its commercial availability and widely investigated properties. ${ }^{[13]}$ Its molecular structure is comprised of a hydrophobic fluoroalkene backbone, to which hydrophilic sulfonated side chains are attached. Upon hydration, Nafion nanosegregates into hydrophobic and hydrophilic subphases. ${ }^{[14]}$ The latter represents a continuous 3D

[*] Dr. J. Landers, ${ }^{[+]}$J. Colon-Ortiz, ${ }^{[+]}$K. Zong, Dr. A. Goswami, Prof. T. Asefa, Prof. A. Vishnyakov, Prof. A. V. Neimark Department of Chemical \& Biochemical Engineering Rutgers, The State University of New Jersey 98 Brett Rd., Piscataway NJ 08854 (USA) E-mail: aneimark@rutgers.edu

$\left.{ }^{+}\right]$These authors contributed equally to this work.

(1) Supporting information for this article can be found under: http://dx.doi.org/10.1002/anie.201606178. network of water domains of the nanometer size. ${ }^{[15,16]}$ An instructive image of nanoscale segregation in a model Nafion film simulated with the coarse-grained dissipative particle dynamics $^{[14]}$ is given in Scheme 1 . The continuity and restricted geometry of the hydrophilic subphase allows for uniform nucleation and controlled growth of MONP. This approach, that has been earlier demonstrated with examples of $\mathrm{Fe}_{2} \mathrm{O}_{3},{ }^{[17]} \mathrm{ZnO},{ }^{[18,19]}$ and from zirconium phosphate, ${ }^{[20,21]}$ has particular advantages over other efforts to incorporate MONP into Nafion, which have largely focused on traditional techniques to fabricate the nanoparticles beforehand then incorporating them into the polymer, such as dropcasting with a polymer resin via the doctor blade method ${ }^{[22]}$ or with the use of organic salts as the precursor. These traditional methods suffer from the disadvantage of larger than desired MONP, and the need for an additional capping agent. Although this method is adept at producing composites, special care is required to thoroughly disperse the nanoparticles throughout, otherwise severe segregation can be anticipated. The method presented herein circumvents this, as the hydrophilic domains responsible for growth are inherently dispersed throughout the polymer. Moreover, the size of the domains naturally limits the size of the nanoparticles, eliminating the need for additional capping agents. In addition, the use of organic salt precursors has focused on hygroscopic materials such as silica $^{[23]}$ and most recently titania. ${ }^{[24]}$ Composite materials fabricated in this fashion have been investigated mainly for fuel cell applications, for instance for improving proton conductivity by increasing water uptake, ${ }^{[25]}$ in reducing methanol cross-over in direct methanol fuel cells, ${ }^{[26-28]}$ increasing the operating temperature of the polymer composite ${ }^{[29]}$ and to a much lesser extent employed in other areas of research for improving vanadium redox battery reactions ${ }^{[30]}$ or for biosensors.${ }^{[31]}$ Notwithstanding these efforts, the library of metals or metal oxides utilized to date is limited (a comprehensive list of those considered have been summarized with their intended purposes in the Supporting Information (SI)). Importantly, there is a lack of understanding how to tailor the size, shape, and distribution of metal oxide nanoparticles within polyelectrolyte membranes.

Herein, we bridge our prior simulation work, ${ }^{[14,32-35]}$ in which we have been able to model and predict the nanostructure segregation in hydrated polyelectrolytes, and leverage this towards designing a robust experimental recipe for in situ fabrication of MONP loaded polyelectrolyte membranes. According to this recipe, impregnation of the hydrated polyelectrolyte films occurs with binary water/ alcohol solutions of metal salts and consecutive reactions that convert metal cations into oxide nanoparticles embedded 
a
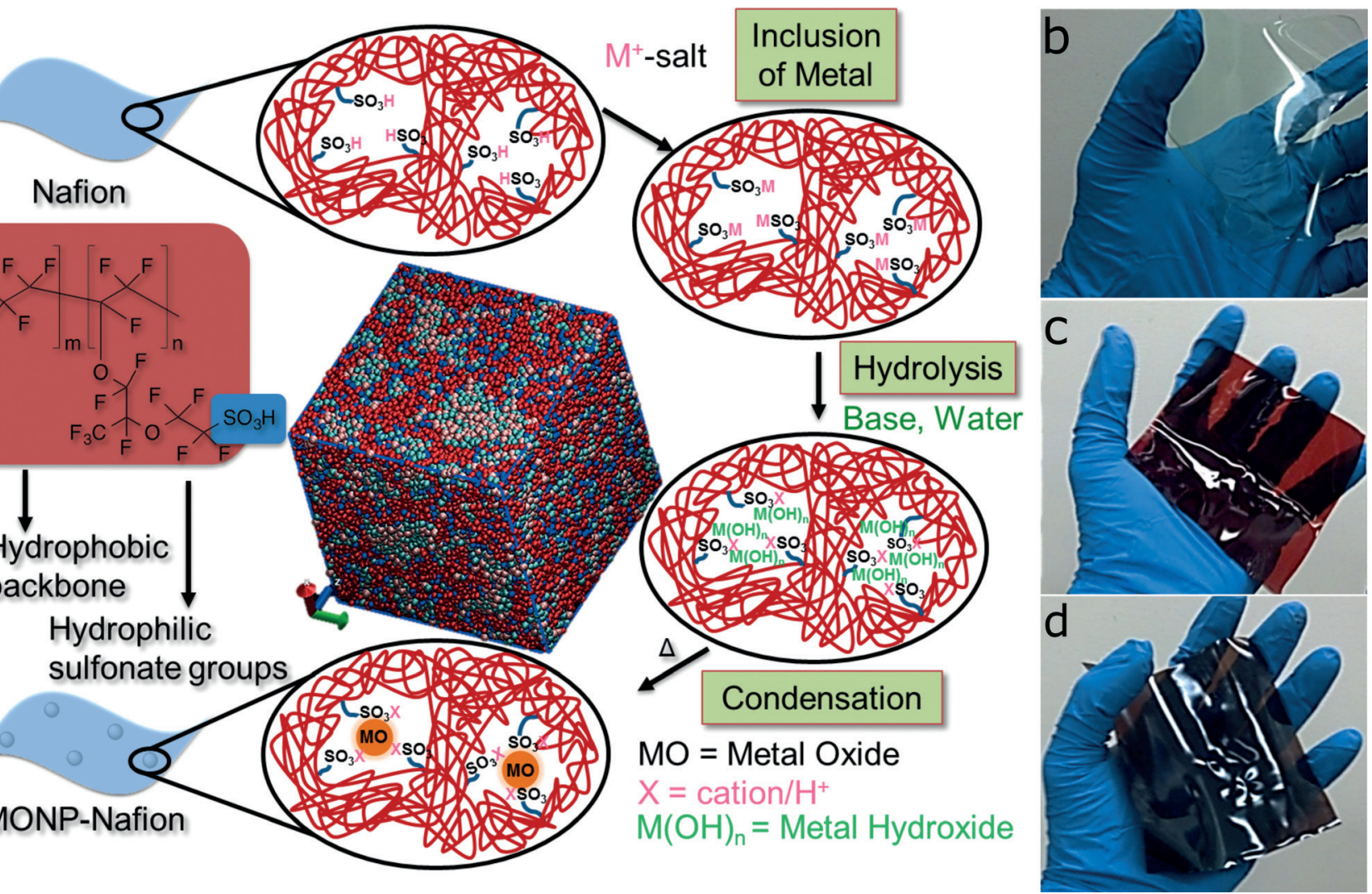

Scheme 1. a) Schematic depicting the in situ growth of MONP within the polyelectrolyte membranes. Centerpiece is a snapshot of the nanoscale segregated structure in a hydrated Nafion membrane produced by the coarse-grained dissipative particle dynamics simulation (red is fluorinated alkene backbone, dark blue is the hydrophilic sulfonate groups, light blue is water, and pink is the counter cation). b-d) Large scale MONPNafion composite films synthesized with nickel oxide, iron oxide and cobalt oxide, respectively.

into the polymer matrix. We show that by varying the metal salt concentration and solvent composition one can affect dispersion, size, and crystallinity of produced nanoparticles, including preferential growth of desired crystal facets. The proposed method is demonstrated with oxides of various metals (nickel, magnesium and cobalt characterization is presented in the SI) focusing on the case study of zinc oxide, one of the earliest and extensively studied MONP systems, whose emission, ${ }^{[36-39]}$ absorption ${ }^{[40]}$ and catalytic ${ }^{[41-43]}$ properties can be finely tuned based on nanoparticle size and shape. The results of this work may have broad implications in the fields of sensing, optoelectronics and catalysis, or wherever there is a need to merge functionality with mechanical flexibility in the form of water permeable, conductive and mechanically robust MONP-polyelectrolyte composite films.

The general scheme of formation of metal oxide nanoparticles (from metal salts) embedded in Nafion film is displayed in Scheme $1 \mathrm{a}$. Specifically for $\mathrm{ZnO}$ nanoparticles the synthesis proceeds as follows: First, a Nafion-117 membrane $(200 \mu \mathrm{m}$ thick with equivalent polymer weight of $1100 \mathrm{Da})$ is impregnated with an aqueous zinc nitrate solution. Zinc(II) ions cluster within the hydrophilic domains via ion exchange with the sulfonated side chains of the Nafion polymer. After allowing the films to saturate with zinc(II) ions, the ionic clusters are converted into the metal form in a strong base $(0.5 \mathrm{M} \mathrm{NaOH})$ at $60^{\circ} \mathrm{C}$. Due to a limited supply of metal ions, the zinc(II) ions continue to hydrolyze to form nanoparticles inside the membrane. At this stage a color change can be observed within the membrane composites due to the formation of zinc hydroxide (Figure S1). Finally, the films are removed from solution and heated at $100{ }^{\circ} \mathrm{C}$ for duration of $24 \mathrm{~h}$ in order to initiate the condensation reaction of the metal hydroxides nanoparticles to metal oxide nanoparticles. Optical images show the synthesized MONPNafion membranes prepared from three different metal nitrate salts (Scheme 1b-d).

Scanning transmission electron microscopy (STEM) confirms the in situ growth of MONP within the hydrophilic domains, evident in Figures $1 \mathrm{a}-\mathrm{d}$. Although the STEM images confirm the presence of particles $<5 \mathrm{~nm}$ in size synthesized from $\mathrm{NaOH}$, higher clarity was achieved for particles synthesized with $\mathrm{NH}_{4} \mathrm{OH}$ revealing the facet planes and hydrophilic domains, and have thus also been included. Dark field images reveal the particles to be dispersed throughout the membrane in some instances separated by no more than $1 \mathrm{~nm}$. Both the $\{002\}$ plane and the $\{100\}$ surface facets of the wurzite structure could be identified (Figure 1c), with the former displaying the expected hexagonal packing arrangement (highlighted in red circles), while the latter displaying the face centered cubic packing. Figures $2 \mathrm{a}$ and $\mathrm{b}$ display lower resolution TEM images of the Nafion membranes prior to and after MONP formation, respectively. The former is characterized by a lack of distinct morphological features, while the latter clearly shows finely dispersed 


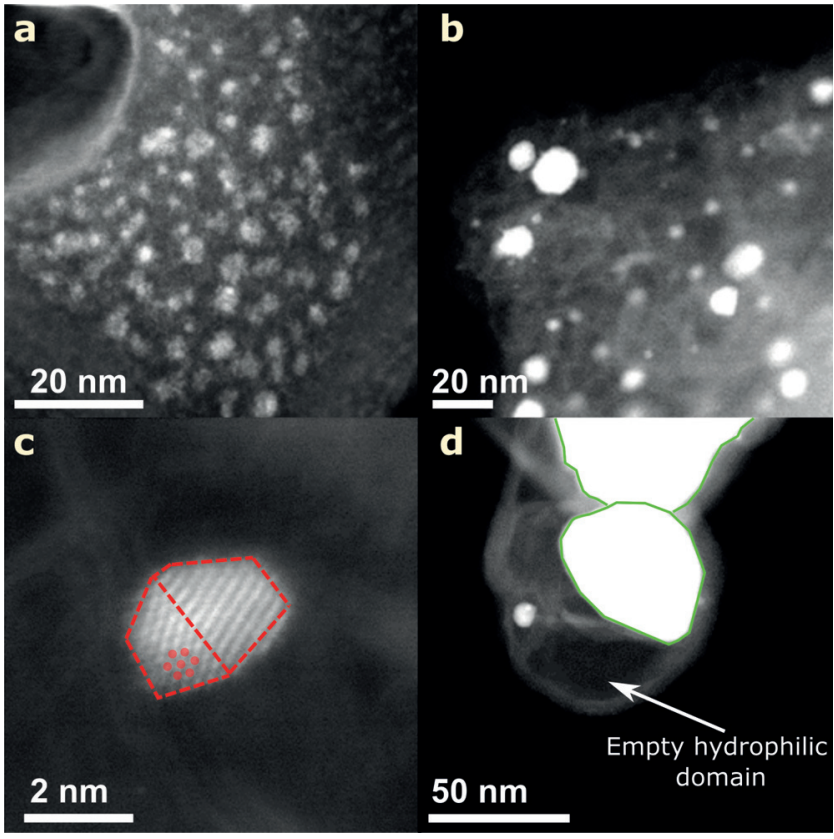

Figure 1. Scanning transmission electron microscopy (STEM) of in situ grown MONP within the polyelectrolyte membrane. a) Dispersed MONP synthesized wtih $\mathrm{NaOH}$. b-d) MONP synthesized using $\mathrm{NH}_{4} \mathrm{OH}$. b) MONP dispersed throughout the Nafion matrix. c) An individual MONP outlined by a dashed red line with the $\{002\}$ and $\{100\}$ exposed crystal facets. The hexagonal packing is highlighted by red circles on the $\{002\}$ plane. d) The host hydrophilic growth domains with MONP grown inside, and evidence of twinning of adjacent MONP, outlined by green.

nanoparticles and their aggregates. Figure $2 \mathrm{c}$ displays SEM/ EDS data revealing the dispersion of zinc (marked in red) to be consistently homogeneous throughout the cross section of the membrane, with higher concentrations of zinc located at the membrane edges. The higher concentrations observed at the edges can be attributed to the inevitable deformation experienced by both sides of the film when cut. This is evident by the lack of deformation that is observed on the edges orthogonal to those observed in Figure 2c (Figure S3). An apparent $\mathrm{Zn}$ concentration in the membrane interior (inside the white frame in Figure $2 \mathrm{c}$ ) is estimated to be $\approx 2 \%$. Independent estimate of the MONP amount was made by thermogravimetric analysis (TGA) shown in the SI (Figure S4), and determines the MONP loading can be as high as $8 \%$, although this should be viewed as an upper limit as the weight will also include contribution from carbon ash. UV-vis measurements were performed in order to estimate the MONP size (Figure 2d). It is known that the absorbance spectra depends on particle size, thus the sharp absorbance onset that is observed is indicative of a narrow distribution. ${ }^{[4]}$ Using the model developed by Pesika et al. ${ }^{[44]}$ the particle size was determined to be $\approx 4 \mathrm{~nm}$, in agreement with the predicted hydrophilic domain size of $3-5 \mathrm{~nm} .{ }^{[14]}$ Within the membranes there appears to be the existence of two different size regimes that varies with solvent composition: nanoparticles and aggregates. Understanding the promotion or prevention of either can therefore be an utmost concern. The lower resolution TEM employed in this study allowed for rapid sampling of different locations in order to identify areas with larger aggregates in addition to a high throughput of different samples, from which a statistical analysis could be performed. The results of which indicates a narrow particle distribution with a mean aggregate size measured to be 10 $20 \mathrm{~nm}$ (Figure 2e and f). These results are not contradictory, as the darker domains appearing in the TEM image displayed in the inset of Figure $2 \mathrm{~b}$ are assumed to be attributed to individually dispersed MONP of $\approx 4 \mathrm{~nm}$ size which could not be quantified in a statistical manner due to the limit of resolution. Noteworthy, the particle size is extraordinarily small for the duration of the 2-step reaction ( $30 \mathrm{~h}$ in total). When the reaction is performed outside the membrane for the same duration, particles on the hundreds of micron scale are formed (see Figure S4). The growth of MONP in Nafion is inhibited by the confining geometry of hydrophilic domains, elastic resistance of the polymer network, as well as by severe diffusion limitations in the hydrophilic subphase.

Wide-angle X-ray diffraction (XRD) performed on the MONP loaded films further confirmed zinc oxide to be of the wurzite form (JCPDS 89-1397). Figure 3 displays the XRD pattern that arises from a typical zinc oxide-Nafion composite. The main features displayed in the pattern are the three prominent peaks located at $2 \theta \approx 31.7^{\circ}, 34.4^{\circ}$ and $36.2^{\circ}$, representing the three most thermodynamically stable crystallographic planes of the wurzite structure (corresponding to the $\{101\},\{002\}$ and $\{100\}$ planes, respectively). The integrated peak areas indicate that under aqueous conditions, the most prominent plane is the $\{101\}$ plane, accounting for $45 \%$ of the total area among the three primary peaks. The integrated peak areas for the $\{100\}$ and $\{002\}$ planes meanwhile each represent approximately $27 \%$. This ratio matches closely with the XRD pattern for the bulk zinc oxide powder (Figure S6) synthesized under the same conditions in solution and is indicative of the presence of zinc oxide nanorod seeds, defined by particles that have the $\{100\}$ facet predominantly exposed, but has not reached the proper aspect ratio that would define a rod-like structure. The XRD patterns are analogous to other studies that have produced zinc oxide particles using various procedures. ${ }^{[45]}$

Binary mixtures of water and four different alcohols (methanol, ethanol, 1-propanol and 2-propanol) were chosen at four different concentrations in order to study the interplay between the swelling behavior of the host polymer and the growth dynamics of the inorganic phase. It was observed that during the in situ growth there was a monotonic increase of swelling with the alcohol content and that swelling is more pronounced for less polar alcohols (Figure S8). This is attributed to the known fact that binary mixtures of alcohol and water results in a greater uptake than their pure components $^{[32]}$ and that the enhanced swelling arises from the preferential solvation of the membrane backbone by the alcohol. ${ }^{[35]}$ TEM image analysis indicate that there is a slight increase in the aggregate size for all conditions containing alcohol producing aggregates sizes much greater than the $12 \mathrm{~nm}$ average that is obtained from aqueous conditions only, possibly arising from the increased swelling of the membrane (Figure $2 \mathrm{f}$ ). In general, the particles synthesized across all concentrations are spatially separated from one another despite the increased swelling, reflecting the heterogeneous 


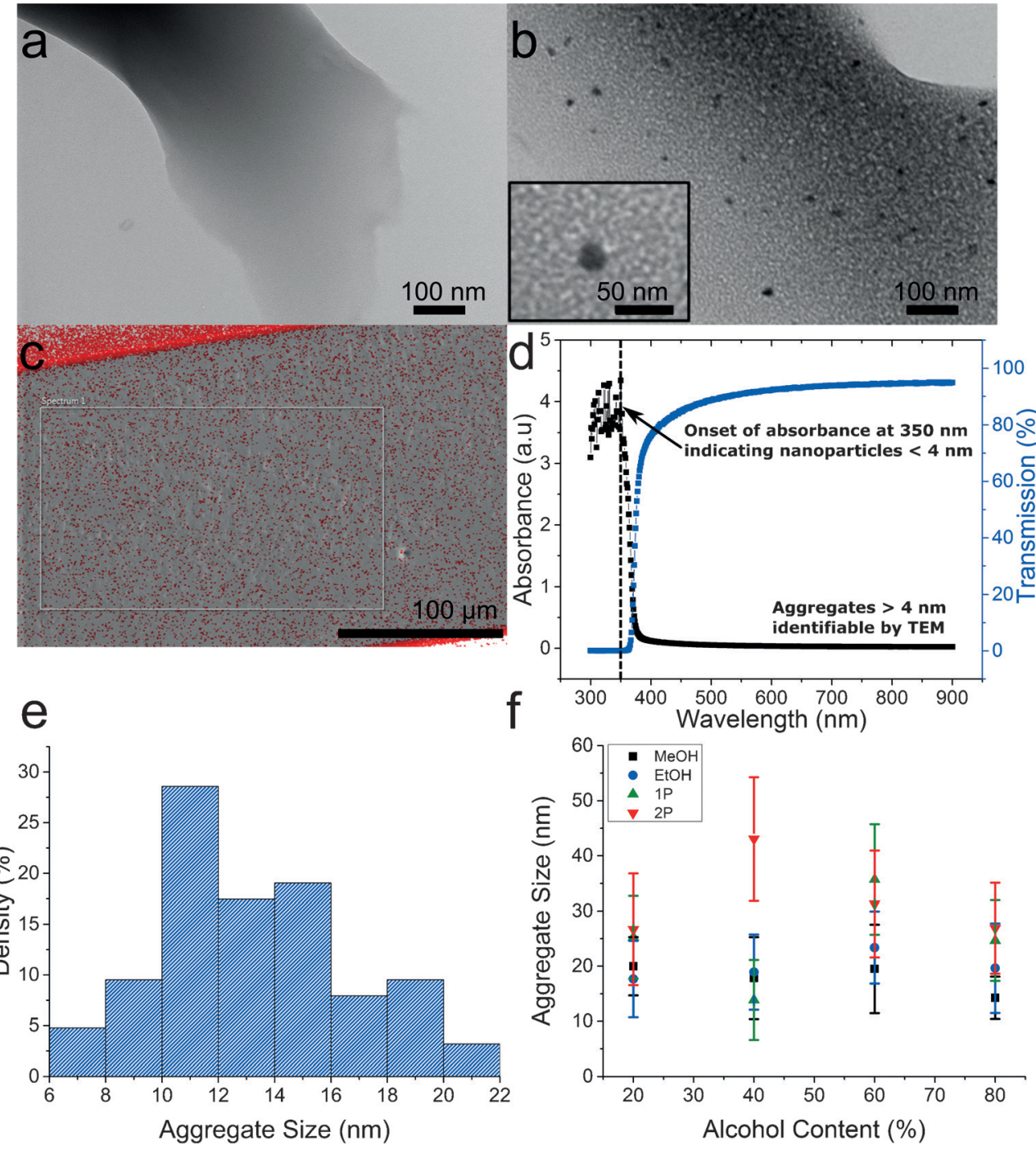

Figure 2. a) TEM image of the parental Nafion film (control sample). b) TEM image of the in situ growth of zinc oxide particles and aggregates within the Nafion film. Inset shows a large aggregate surrounded by finely dispersed individual MONPs. c) SEM/EDS cross section of the zinc oxide-Nafion composite film depicting uniform distribution of $\mathrm{Zn}$ rich (red) regions. d) Absorbance (black) and transmission (blue) measurements for estimating the MONP size. Onset of absorbance at $350 \mathrm{~nm}$ corresponds to the characteristic MONP size of $4 \mathrm{~nm}$. e) Size distribution of MONP aggregates ranging between 6 and $20 \mathrm{~nm}$ derived from TEM images. The image resolution limits the quantification capability by $6 \mathrm{~nm}$ and, thus, this distribution does not include individual MONPs of smaller sizes. f) Average aggregate size as a function of alcohol content and type of alcohol.

morphology of the hydrophilic subphase (Scheme 1a), which prevents the formation of a percolated inorganic phase throughout the membrane. Nonetheless, when higher alcohol concentrations are employed, instances of particle twinning grown in adjacent hydrophilic domains can be observed (Figure 1d and Figure S9).

XRD patterns and fitted peak areas for each condition are displayed in Figure 4. XRD analysis reveals that with the inclusion of any of the 4 alcohols, the peak representing the $\{101\}$ plane of zinc oxide is no longer the most prominent but instead becomes subsidiary to the peak representing the $\{002\}$ crystal plane. The insets for Figure 4 show the evolution of the fitted peak areas for each condition. With increasing alcohol content there is a non-monotonic increase in the $\{002\}$ plane that exceeds the growth of both the $\{101\}$ and $\{100\}$ planes, which corresponds to the formation of zinc oxide nanoplatelets. ${ }^{[45]}$ Classic nucleation theory states that energetically favored crystalline planes will be promoted at the expense of less thermodynamic stable ones. ${ }^{[46]}$ By varying the solvent both the nucleation rate and growth kinetics can be altered in such way that can lead to the rise of otherwise less stable crystalline planes. Interestingly, when zinc oxide is synthesized in the bulk solution (i.e. without the membrane) the integrated peak areas conform back to the ratio ascribed to the formation of zinc oxide without the presence of alcohol (i.e. the most prominent plane is the $\{101\}$, Figure S6). This holds true for the longest alkyl chain alcohol used in this study, that is, 2-propanol, at all concentrations (Figure S7). This is not surprising as the most common capping agents on zinc oxides are polymers and amines, not alcohols, and ripening behavior will lead to more thermodynamically stable facets overtaking less stable ones at larger particle sizes. $^{[46]} \quad$ Furthermore, a large local concentration of alcohols within the Nafion membrane may also impart a significant role in directing facet growth that is not possible during the bulk growth. Notably, the formation of zinc oxide within the membranes does not occur without the presence of water (Figure S10), that is, when only alcohol is used. This is an obvious conclusion as water is critical for the formation of the metal hydroxides during hydrolysis. Changes in the hydrophilic domain structure may explain the preferred growth of the $\{002\}$ plane of zinc oxide in alcoholic solutions. Within a given domain the concentration and proximity of the sulfonated groups are assumed to be fixed. However, microenvironmental changes related to the membrane swelling may produce de facto changes that create a less dense packing of sulfonated groups within the hydrophilic domain that promotes the growth of the $\{002\}$ plane. Moreover, Wang et al. 


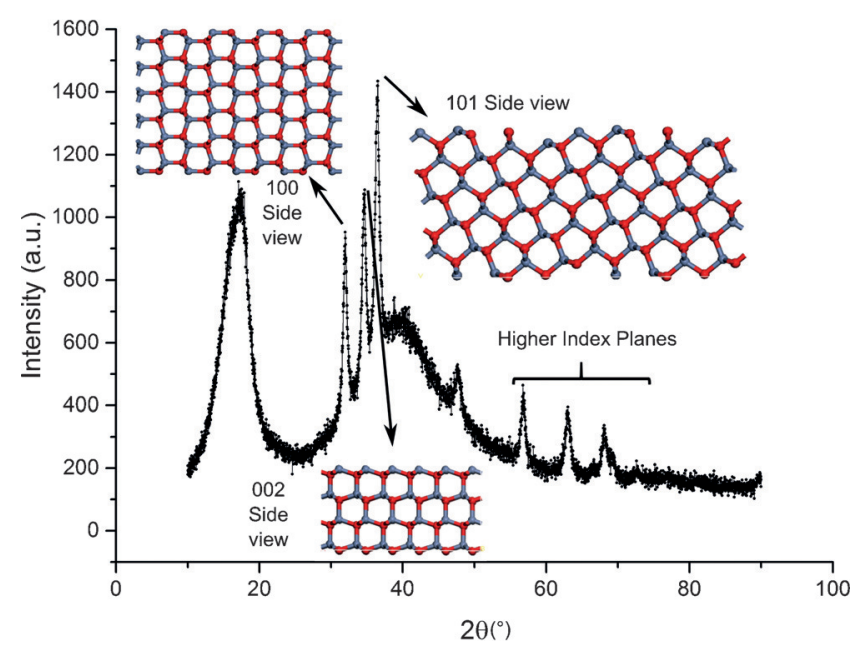
grown within the Nafion film under aqueous conditions. The pattern exhibits the wurzite form of zinc oxide with the three primary peaks (indicated by arrows) representing the $\{100\},\{002\}$ and $\{101\}$ crystalline planes.
Figure 3. X-ray diffraction (XRD) pattern for zinc oxide nanoparticles

have suggested that when ethanol is used in the hydrolysis step, a greater amount of $\mathrm{OH}^{-}$groups cluster in the hydrophilic cavities, leading to a plethora of oxygen defects. ${ }^{[19]}$ This in turn led to a greater enhancement of photocatalytic capability of their composite membrane.

As previously mentioned, the use of less polar alcohols resulted in a tendency to form larger zinc oxide aggregates exacerbated by higher alcohol contents. This increase in aggregate size is accompanied by a decrease in intensities for the higher index peaks (i.e. corners and edges) that appear in the XRD pattern (Figure S11). By comparing relative peak intensities for all peaks, it can be observed that the use of higher homologous alcohols led to a decrease in total intensities for the higher index planes. For example, the higher index peaks amounted to over $30 \%$ of the total peak intensity for all concentrations of methanol. This is contrary to the case of 2-propanol where the accumulated peak intensity amounted to $20 \%$ of the total. Thus it could be envisioned that although the $\{002\}$ plane is more catalytically active in certain scenarios over the $\{101\}$ plane, it comes at the consumption of highly active corners and edges.

In conclusion, we investigated the conditions that lead to the in situ generation of MONPs within polyelectrolyte membranes.
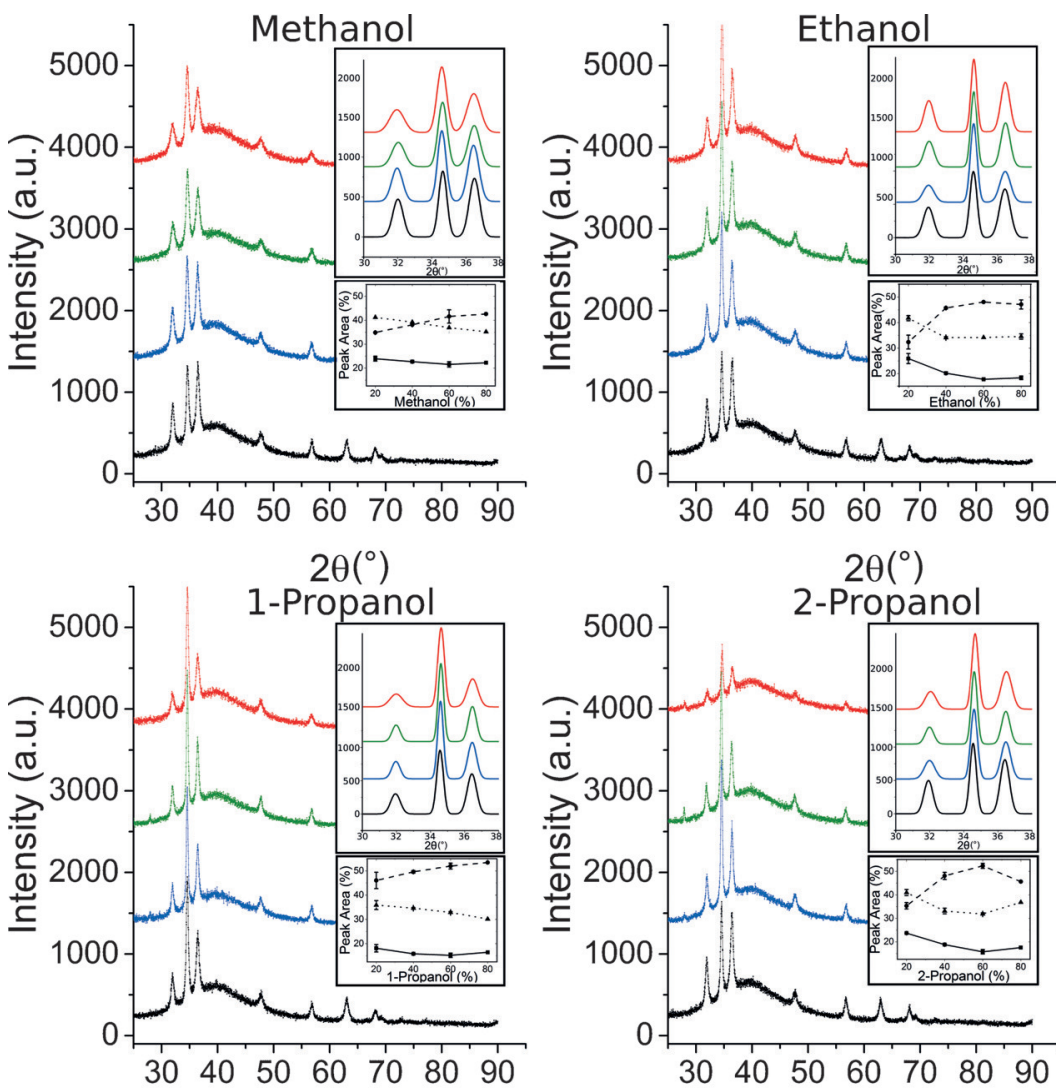

$2 \theta\left(^{\circ}\right)$

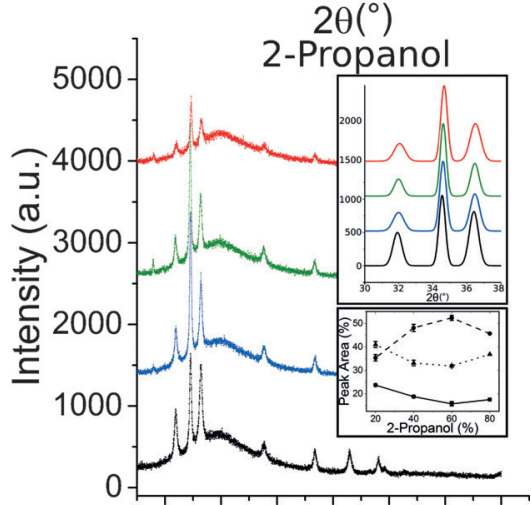

$\begin{array}{llllllll}30 & 40 & 50 & 60 & 70 & 80 & 90\end{array}$

$2 \theta\left(^{\circ}\right)$
Figure 4. Wide-angle X-ray diffraction for zinc oxide-Nafion composites prepared with different binary solvent mixtures. The percent of alcohol in the binary mixtures are $20 \%$ (black), $40 \%$ (blue) $60 \%$ (green) and $80 \%$ (red). Top inset includes the fitted data for the low index planes corresponding to composites prepared at different concentrations of alcohol. Y-axis displays the intensity. The bottom inset plots the evolution of the three crystalline planes as a function of alcohol content. The lines correspond to the following planes: $-\{100\},---\{002\}$ and $\cdots . . .\{101\}$.
The method was demonstrated drawing on the example of Nafion membranes and a variety of metal oxides with an emphasis placed on zinc oxide. The STEM, TEM, SEM/EDS, UV-vis and XRD studies confirmed the homogeneous distribution within the membrane of crystalline MONP of $\approx 4 \mathrm{~nm}$ and their aggregates of $10-20 \mathrm{~nm}$. It was shown that the size and morphology of in situ grown MONPs can be controlled through the use of binary water-alcohol solvents of different composition. Meanwhile, the inclusion of alcohol in the solvent promoted the dominant growth of the $\{002\}$ plane of zinc oxide and thus the formation of nanoplatelets as opposed to nanorods. This is contrary to ex situ growth conditions, when the $\{002\}$ plane is consigned to being the least prom$\{101\})$. The ability to tailor the MONP size and crystallinity during the in situ growth has far reaching implications in catalysis and optoelectronics, as well as in applications for sensors and chemical protection. In particular, since MONPs, such as $\mathrm{ZnO}$, are known to catalyze decomposition of chemical warfare agents and industrial toxic chemicals ${ }^{[47-49]}$ and the polyelectrolyte films, like Nafion, are used as diffusion barriers, ${ }^{[32,50,51]}$ the polyelectrolyte films loaded with catalytically active MONPs may constitute the foundation for highly sought self-detoxificating protective membranes. ${ }^{[52]}$ inent of the 3 surface planes ( $\{100\},\{002\}$, 


\section{Acknowledgements}

This work was supported by DTRA grant HDTRA1-14-10015 .

Keywords: composite membranes - Nafion . proton exchange membranes · self-assembly

How to cite: Angew. Chem. Int. Ed. 2016, 55, 11522-11527 Angew. Chem. 2016, 128, 11694-11699

[1] S. Sarkar, E. Guibal, F. Quignard, A. K. SenGupta, J. Nanopart. Res. 2012, 14, 24.

[2] Z. Weng, W. Liu, L. C. Yin, R. P. Fang, M. Lo, E. I. Altman, Q. Fan, F. Li, H. M. Cheng, H. L. Wang, Nano Lett. 2015, 15, 7704

[3] R. Schlögl, Angew. Chem. Int. Ed. 2015, 54, 3465; Angew. Chem. 2015, 127, 3531 .

[4] A. Tricoli, M. Righettoni, A. Teleki, Angew. Chem. Int. Ed. 2010, 49, 7632; Angew. Chem. 2010, 122, 7796.

[5] D. Pradhan, F. Niroui, K. T. Leung, ACS Appl. Mater. Interfaces 2010, 2, 2409.

[6] A. R. Hopkins, N. S. Lewis, Anal. Chem. 2001, 73, 884.

[7] S. Sundarrajan, S. Ramakrishna, J. Mater. Sci. 2007, 42, 8400.

[8] S. Rosset, H. R. Shea, Appl. Phys. A 2013, 110, 281.

[9] L. Wang, M. H. Yoon, G. Lu, Y. Yang, A. Facchetti, T. J. Marks, Nat. Mater. 2006, 5, 893.

[10] C. Fenzl, T. Hirsch, O. S. Wolfbeis, Angew. Chem. Int. Ed. 2014, 53, 3318; Angew. Chem. 2014, 126, 3384.

[11] C. Z. Yuan, H. B. Wu, Y. Xie, X. W. Lou, Angew. Chem. Int. Ed. 2014, 53, 1488; Angew. Chem. 2014, 126, 1512.

[12] S. Sundarrajan, A. R. Chandrasekaran, S. Ramakrishna, J. Am. Ceram. Soc. 2010, 93, 3955.

[13] M. Rikukawa, K. Sanui, Prog. Polym. Sci. 2000, 25, 1463.

[14] A. Vishnyakov, A. V. Neimark, J. Phys. Chem. B 2014, 118, 11353.

[15] X. Ling, M. Bonn, S. H. Parekh, K. F. Domke, Angew. Chem. Int. Ed. 2016, 55, 4011; Angew. Chem. 2016, 128, 4079.

[16] J. Song, O. H. Han, S. Han, Angew. Chem. Int. Ed. 2015, 54, 3615 ; Angew. Chem. 2015, 127, 3686.

[17] L. Raymond, J. F. Revol, D. H. Ryan, R. H. Marchessault, J. Appl. Polym. Sci. 1996, 59, 1073.

[18] H. Wang, P. Liu, S. Wang, W. Han, X. Wang, X. Fu, J. Mol. Catal. A 2007, 273, 21.

[19] J. Wang, P. Liu, X. Fu, Z. Li, W. Han, X. Wang, Langmuir 2009, $25,1218$.

[20] M. L. Hill, Y. S. Kim, B. R. Einsla, J. E. McGrath, J. Membr. Sci. 2006, 283, 102

[21] M. K. Song, J. S. Hwang, Y. T. Kim, H. W. Rhee, J. Kim, Mol. Cryst. Liq. Cryst. 2003, 407, 421.

[22] M. A. Harmer, W. E. Farneth, Q. Sun, J. Am. Chem. Soc. 1996, 118, 7708.

[23] K. A. Mauritz, D. A. Mountz, D. A. Reuschle, R. I. Blackwell, Electrochim. Acta 2004, 50, 565.

[24] M. Amjadi, S. Rowshanzamir, S. J. Peighambardoust, M. G. Hosseini, M. H. Eikani, Int. J. Hydrogen Energy 2010, 35, 9252.
[25] S. J. Peighambardoust, S. Rowshanzamir, M. Amjadi, Int. J. Hydrogen Energy 2010, 35, 9349.

[26] Y. Daiko, L. C. Klein, T. Kasuga, M. Nogami, J. Membr. Sci. 2006, 281, 619.

[27] D. H. Jung, S. Y. Cho, D. H. Peck, D. R. Shin, J. S. Kim, J. Power Sources 2002, 106, 173.

[28] B. P. Ladewig, R. B. Knott, D. J. Martin, J. C. D. da Costa, G. Q. Lu, Electrochem. Commun. 2007, 9, 781.

[29] Q. Deng, C. A. Wilkie, R. B. Moore, K. A. Mauritz, Polymer 1998, 39, 5961.

[30] X. G. Teng, Y. T. Zhao, J. Y. Xi, Z. H. Wu, X. P. Qiu, L. Q. Chen, J. Membr. Sci. 2009, 341, 149.

[31] E. J. Choi, C. H. Kang, H. N. Choi, W. Y. Lee, Bull. Korean Chem. Soc. 2009, 30, 2387.

[32] D. Rivin, G. Meermeier, N. S. Schneider, A. Vishnyakov, A. V. Neimark, J. Phys. Chem. B 2004, 108, 8900.

[33] A. Vishnyakov, A. V. Neimark, J. Phys. Chem. B 2000, 104, 4471.

[34] A. Vishnyakov, A. V. Neimark, J. Phys. Chem. B 2001, 105, 9586.

[35] A. Vishnyakov, A. V. Neimark, J. Phys. Chem. B 2001, 105, 7830.

[36] L. Qian, Y. Zheng, J. G. Xue, P. H. Holloway, Nat. Photonics 2011, 5, 543 .

[37] J. H. Zhang, B. D. Zhao, Z. D. Pan, M. Gu, A. Punnoose, Cryst. Growth Des. 2015, 15, 3144.

[38] B. L. Greenberg, S. Ganguly, J. T. Held, N. J. Kramer, K. A. Mkhoyan, E. S. Aydil, U. R. Kortshagen, Nano Lett. 2015, 15, 8162.

[39] R. Zamiri, A. Zakaria, H. A. Ahangar, M. Darroudi, A. K. Zak, G. P. C. Drummen, J. Alloys Compd. 2012, 516, 41.

[40] S. B. Khan, M. Faisal, M. M. Rahman, A. Jamal, Talanta 2011, 85, 943.

[41] P. Fageria, S. Gangopadhyay, S. Pande, RSC Adv. 2014, 4, 24962.

[42] G. Ramakrishna, H. N. Ghosh, Langmuir 2003, 19, 3006.

[43] O. Z. Didenko, G. R. Kosmambetova, P. E. Strizhak, J. Mol. Catal. A 2011, 335, 14.

[44] N. S. Pesika, K. J. Stebe, P. C. Searson, Adv. Mater. 2003, 15, 1289.

[45] Y.-K. Peng, L. Ye, J. Qu, L. Zhang, Y. Fu, I. F. Teixeira, I. J. McPherson, H. He, S. C. Edman Tsang, J. Am. Chem. Soc. 2016 , $138,2225$.

[46] T. L. Sounart, J. Liu, J. A. Voigt, M. Huo, E. D. Spoerke, B. McKenzie, J. Am. Chem. Soc. 2007, 129, 15786.

[47] T. H. Mahato, G. K. Prasad, B. Singh, J. Acharya, A. R. Srivastava, R. Vijayaraghavan, J. Hazard. Mater. 2009, 165, 928.

[48] G. K. Prasad, T. H. Mahato, B. Singh, K. Ganesan, P. Pandey, K. Sekhar, J. Hazard. Mater. 2007, 149, 460

[49] V. Houšková, V. Štengl, S. Bakardjieva, N. Murafa, A. Kalendová, F. Opluštil, J. Phys. Chem. A 2007, 111, 4215.

[50] M. T. Lee, A. Vishnyakov, G. Y. Gor, A. V. Neimark, J. Phys. Chem. B 2013, 117, 365.

[51] M. T. Lee, A. Vishnyakov, G. Y. Gor, A. V. Neimark, J. Phys. Chem. B 2011, 115, 13617.

[52] S. Sundarrajan, A. Venkatesan, S. Ramakrishna, Macromol. Rapid Commun. 2009, 30, 1769.

Received: June 25, 2016

Published online: August 19, 2016 